\title{
Impact of Form Factors and Input Conditions on Absolute Indirect-Touch Pointing Tasks
}

\author{
Jérémie Gilliot \\ Inria Lille, France \\ jeremie.gilliot@inria.fr
}

\author{
Géry Casiez \\ University of Lille, France \\ gery.casiez@lifl.fr
}

\author{
Nicolas Roussel \\ Inria Lille, France \\ nicolas.roussel@inria.fr
}

\begin{abstract}
Absolute indirect interaction maps the absolute position of a device's end-effector to the absolute position of a remote onscreen object. Despite its long-time use with graphics tablets and growing use in research prototypes, little is known on the influence of form factors and input conditions on pointing performance with such a mapping. The input and display can have different sizes and aspect ratios, for example. The on-screen targets can vary in size. Users can look solely at the display or at the input device as well. They can also hold the input device in certain cases, or let it rest on a table. This paper reports on two experiments designed to investigate the influence of all these factors on absolute indirect-touch pointing performance. We also provide design guidelines for interaction in these situations based on the observed impacting factors.
\end{abstract}

\section{Author Keywords}

Indirect touch; absolute pointing; performance; form factors; input conditions

\section{ACM Classification Keywords}

H.5.2 Information interfaces and presentation: User interfaces

\section{INTRODUCTION}

Touch-based interactions use either an absolute or a relative mapping. The relative one maps the displacement of a contact on the input surface to that of an on-screen object. It generally uses a non-linear transfer function to support fast movements over large distances and precise interactions with small objects [2]. In contrast, an absolute mapping establishes an homothetic correspondence between the position of a contact on the input surface and that of an on-screen object. A mapping will be said to be direct when the touched surface and the screen are co-localized. A relative direct mapping is quite unusual, but possible [4]. Smart phones normally use an absolute direct mapping and touchpads a relative indirect one. Graphics tablets often support both a relative indirect and an absolute indirect mapping. Absolute indirect mappings have also been used in various research prototypes.

Permission to make digital or hard copies of all or part of this work for personal or classroom use is granted without fee provided that copies are not made or distributed for profit or commercial advantage and that copies bear this notice and the full citation on the first page. Copyrights for components of this work owned by others than ACM must be honored. Abstracting with credit is permitted. To copy otherwise, or republish, to post on servers or to redistribute to lists, requires prior specific permission and/or a fee. Request permissions from Permissions@ acm.org.

CHI'14, April 26-May 01 2014, Toronto, ON, Canada

Copyright 2014 ACM 978-1-4503-2473-1/14/04 . .\$15.00.

http://dx.doi.org/10.1145/2556288.2556997
The absolute direct mapping of many multi-touch interfaces contributes to a high feeling of directness [10], as it provides the illusion of touching the manipulated objects. But it can make small targets difficult to acquire and fatigue can quickly set in with large screens, due to important distances to travel, or vertical ones. Relative indirect multi-touch interaction could help alleviate these problems, but it requires the control of several remote pointers at the same time, a difficult task despite attempts at improving it $[15,5,1]$.

Absolute indirect mappings support easier multi-touch interaction at a distance, especially when combined with specific feedback. Graphics tablets usually provide hovering information allowing the visual representation of imminent contacts, for example. Most multi-touch systems do not, however. A camera mounted above the input surface is thus sometimes used to remotely display a silhouette of the arms and fingers $[13,17]$. Absolute indirect mappings have been used for the control of multiple cursors $[15,1]$, interaction with large screens $[13,14,16]$ and on-body interaction $[12,6,7]$. But in each of these works, the characteristics and configuration of the input and output surfaces were fixed and determined for unknown reasons. Little is actually known about the influence of input and output parameters on users' ability to manipulate or simply point at objects in these situations. Most commercial touchpads provide absolute finger position information, even if it is most commonly used to compute relative displacements. Absolute indirect interaction is thus not bound to hardware limitations or hardware availability, but to the development of proper knowledge and interaction techniques.

This paper reports on two experiments investigating the influence of form factors of the input device (size and aspect ratio), form factors of the display (device size and aspect ratio, target size) and input conditions (ability to look at the device, ability to use both hands) on participants' performance in absolute indirect-touch pointing tasks. We also provide design guidelines for interaction in these situations based on the observed impacting factors.

\section{RELATED WORK}

We first describe some uses of absolute indirect-touch pointing and then discuss studies of the factors impacting performance in these situations.
Absolute Indirect-Touch Pointing
An absolute indirect mapping has been proposed by McCal- lum and Irani for coarse cursor positioning on a large display using a mobile phone [14]. Their ARC-Pad technique actu- ally combines an absolute and a relative mapping: the user 
taps on the phone's screen to set the remote cursor's initial location in absolute mode, then drags the finger to adjust it in a relative mode. The authors report that ARC-Pad's absolute positioning reduced clutching by half when compared to relative positioning alone (using Windows XP's cursor acceleration). This suggests a good level of performance for participants in absolute indirect pointing tasks. But as the technique was evaluated with a single input device and a single screen, it is unclear whether their characteristics played a part in participants' performance.

To avoid large scaling effects when interacting in absolute mode on a large screen with a small input surface, a solution is to map the latter to only a sub-region of the former. Malik et al. followed this approach with a $500 \times 180 \mathrm{~cm}$ display and a $60 \times 20 \mathrm{~cm}$ custom-made touchpad with remote video feedback [13]. The left half of the touchpad was mapped to the whole screen and allowed to control the absolute position of an on-screen delimited workspace. The right half was mapped to the workspace, allowing finer absolute interaction inside it. The workspace was set to a size "such that every pixel on the display can be reached using a combination of coarse and fine positioning" and could be resized. Some users reportedly felt that the default precision on the right side was too coarse. But the impact of the sizes of the manipulated objects and input and display surfaces was not investigated.

Indirect multi-touch cursors have been proposed for touchpads and mice that allow the relative positionning of the cursor space on the display and the absolute positioning of cursors within $[15,1]$. In both cases, however, nothing was said about how the cursor space should be defined, its relation to the size of the input surface, the characteristics of the task or users' capabilities.

Absolute indirect mappings have also been used for screenless imaginary interfaces, where people point in empty space or onto their own body. Gustafson et al. showed that people can build spatial memory using a real phone that transfers to an imaginary one and discussed various mappings between the phone's screen and the non-dominant palm [6]. But both have relatively similar sizes, and it is unclear whether spatial memory would have transferred if it was not the case.

\section{Factors Impacting Performance}

Wigdor et al. studied the effects of display position and control space orientation on user preference and performance in absolute indirect docking tasks [21]. Participants predominantly preferred a display space location offset $45^{\circ}$ from a facing position (NW or NE instead of $\mathrm{N}$ ). The most suitable orientation of input space varied with screen position. For front screen positions (NW, N, NE), best performance was obtained with a $45^{\circ}$ offset from straight-on in controlorientation.

Schmidt et al. compared the performance of absolute direct and indirect mappings for multi-touch docking tasks on a custom $100 \mathrm{~cm}$ (diagonal) DI table [17]. The indirect mapping used video feedback to show hand contours and circles as feedback for contacts. Completion times were found to be shorter and error rates lower in the direct condition. But half of the participants complained it was difficult to coordinate their hands with the remote video feedback. This, according to the authors, might partially explain the observed degradation of performance.

Most studies on touch accuracy were conducted using an absolute direct mapping. An early study from Hall et al. on an IBM InfoWindow touchscreen reported that accuracy varied from $66.7 \%$ for $10 \mathrm{~mm}$ targets to $99.2 \%$ for $26 \mathrm{~mm}$ ones [8]. In their study of the Shift technique on a PDA, Vogel and Baudisch found a minimum size for reliable acquisition of $10.5 \mathrm{~mm}$ [19]. In another study on a FTIR table, Wang et al. found $11.5 \mathrm{~mm}$ [20]. Using a FingerWorks iGesture touchpad, Holz and Baudisch found $15 \mathrm{~mm}$ [9]. In the specific context of touch typing, studies showed that participants favored and were more efficient with keys larger than $19 \mathrm{~mm}$, the size typically used for physical keyboards [18, 3].

Touch accuracy has also been studied in screen-less absolute indirect mappings. Lin et al. studied target selection along a one-dimensional axis on the forearm [12]. Participants were asked to touch one of 5 to 9 points between the wrist and elbow of their non-dominant arm (typically $22 \mathrm{~cm}$ long). The setting prevented them from seeing their arms while doing so. Participants were able to acquire 6 different points, and exceptionally 8. Gustafson et al. studied accuracy in 2D imaginary tapping tasks [6]. Participants were asked to tap on their non-dominant hand or in an empty space beside it at a position learnt on an iPod Touch. The minimum size for reliable acquisition was $27.9 \mathrm{~mm}$ for the empty space and $17.7 \mathrm{~mm}$ for the palm. In a subsequent study, tapping was replaced with a browsing task [7]. Participants were asked to search and select a target by moving a finger accross their palm, the name of hovered targets being audibly announced. Focusing on selection time, the authors found that the ability to see the hands outperforms the tactile cues they provide. When blindfolded, the tactile cues from the palm were more important than those from the fingertip. Pietroszek and Lank used separated input and output surfaces with the same aspect ratio (4:3) and targets of different sizes always visible on the output surface and either visible or not on the input one. They found a high level of accuracy for targeting without visual cues on the input surface, which was quite surprising to them, and a two-fold increase in accuracy with them. They acknowledge in [16] that form factors can affect performance and that "additional evaluation is needed for non-matching aspect ratio".

To summarize, previous works have largely ignored the potential influence of the form factors of the input device and display on absolute indirect-touch pointing performance. Knowledge on this matter could help better understand the performance observed with techniques based on such a mapping. It could also help adjusting some of their parameters, such as the dimensions of a virtual workspace $[13,15,1]$.

In what follows, we report on two experiments on absolute indirect-touch pointing. The first one focused on the influence of the input device size and other input conditions on performance, while the second one focused on scale effects and aspect ratio. The same task was used in the two exper- 
iments. Participants were shown a target on a display and asked to point at the corresponding location on a separate touch-enabled surface using the index finger of their dominant hand. No artificial feedback of any kind was provided to help them in this task. We did not include a condition where the targets would be displayed on the input surface, as the task would then reduce to an absolute direct mapping for which targeting accuracy has already been studied in the literature.

\section{EXPERIMENT 1: DEVICE SIZE AND INPUT CONDITIONS}

The goal of this experiment was to evaluate how the size of the input device affects the minimum target size users can successfully acquire on first attempt. We hypothesized that despite the lack of visual feedback on this device, looking at it would help position the index finger. Based on [7], we also hypothesized that the non-dominant hand could act as a reference frame to position the finger if users were unable to look at the device.

\section{Apparatus}

We used two input devices. The first was an iPad 1 featuring a $196 \times 147 \mathrm{~mm}$ input surface. The second was an iPod Touch 3 whose input surface was downsized to $66 \times 50 \mathrm{~mm}$ using a cardboard overlay to have the same $4: 3$ aspect ratio as the iPad (Figure 1, left). The screen of these devices was kept blank white during the experiment which ran on a 2007 MacBook Pro (OS X 10.7, 2.4 GHz, 4 GB of RAM) with a 17.2" LCD display $(367 \times 229 \mathrm{~mm}, 1680 \times 1050$ pixels $)$.

The laptop was set up on a desk in front of the participant, its screen tilted $30^{\circ}$ backward about $60 \mathrm{~cm}$ away from the eyes. The input device (iPad or iPod) was placed on the laptop's palm rest, about $50 \mathrm{~cm}$ away from the eyes. The thickness of the palm rest made it possible to hold the input device in place with the non-dominant hand, without moving it. The software used was coded in $\mathrm{C}++$ using the Qt toolkit. TUIO was used over UDP to send touch events from the input devices to the laptop, the three of them being connected to the same wireless network. Custom-made blinder glasses were also used in certain conditions to prevent participants from seeing their hands and the input device (Figure 1, right).

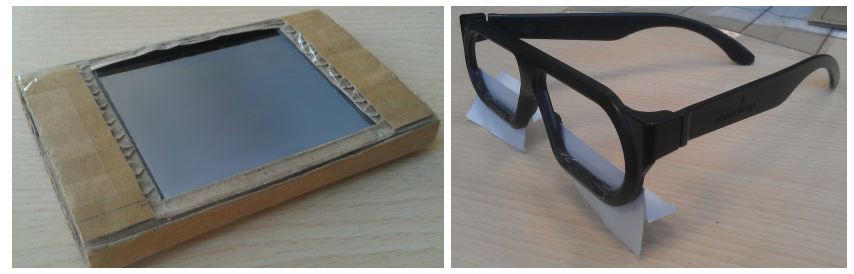

Figure 1: The cardboard overlay covering the iPod Touch (left) prevented touch detection outside the open area. The blinder glasses (right) were made from plastic ones by taping pieces of paper at their bottom.

\section{Task and procedure}

We used a discrete 2D pointing task. Each trial began with a new target appearing on-screen and ended with its successful selection, by touching the input surface at the corresponding location and lifting the finger, or after 5 failed attempts. Every time a participant missed a target, an error was logged.

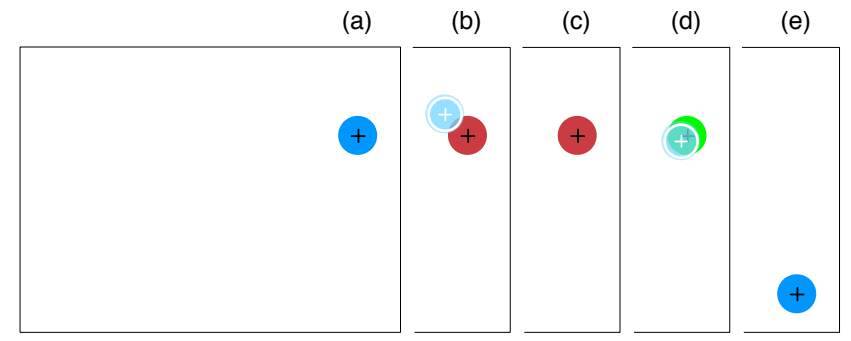

Figure 2: Possible image sequence for a trial: (a) the trial starts with a target to acquire; (b) contact is detected on the input surface, but the attempt failed; (c) contact is lost, the trial continues with a new attempt; (d) contact is detected again and this time, the target was successfully acquired; (e) contact is lost, which initiates the next trial. This particular example would be marked as an error since the target was not successfully acquired on first attempt.

The laptop's screen showed a black background with a white rectangular workspace the same size (in $\mathrm{mm}$ ) as the white input surface. Within this workspace, the target to acquire was shown as a blue disk with a black cross-hair at its center (Figure 2, a). Participants were asked to point on the input surface at the location corresponding to the cross-hair. Touching the input surface resulted in the display of a mark at the corresponding screen location, a semi-transparent blue disk with a white cross hair at its center (b). This mark remained visible as long as contact was detected, but it could not be moved. Missed targets turned red and there was no way of correcting the selection other than retrying (c). Targets successfully acquired turned green (d).

After each block of trials, participants were encouraged to take a break before moving to the next one by pressing a button. Participants were instructed to balance speed and accuracy: they were asked to do their best to select targets while keeping quick and relaxed gestures.

\section{Participants}

12 unpaid volunteers served in the experiment: 4 female and 8 male, all right-handed, with a mean age of $25.5(\mathrm{SD}=1.9)$.

\section{Design}

A repeated measures within-subjects design was used. The independent variables were the input device size (DevicE SizE), the input condition (INPUT CONDITION), and the target position (TARGET POSITION) and size (TARGET SIZE).

Device size was evaluated with 2 levels (SMAll for the iPod, and LARGE for the iPad). TARGET SIZE was evaluated with 3 levels consistent with the minimum target sizes found in the literature $(\mathrm{Ws}=10 \mathrm{~mm}, \mathrm{WM}=20 \mathrm{~mm}$ and $\mathrm{WL}=40 \mathrm{~mm})$. TARGET POSITION was evaluated with 9 levels corresponding to selected intersections of an imaginary $5 \times 5$ grid centered on-screen and numbered from top to bottom and left to right $(1,3,9,10,11,13,19,22,25$ on Figure 3$)$. These positions were limited to 9 to shorten the duration of the experiment but nonetheless chosen to cover at best the entire input surface.

INPUT CONDITION was evaluated with 3 levels: 1HAND, 1HANDBLINDERS and 2HANDSBLINDERS (Figure 4). In the 1HAND condition, participants could but were not enforced to look at the input surface. In 1HANDBLINDERS and 2HANDSBLINDERS, they 


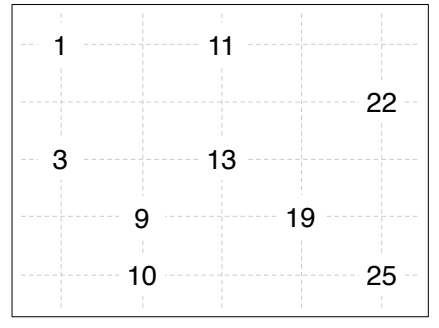

Figure 3: Locations used for the TARGET POSITION variable (the grid and numbers were not shown to participants).

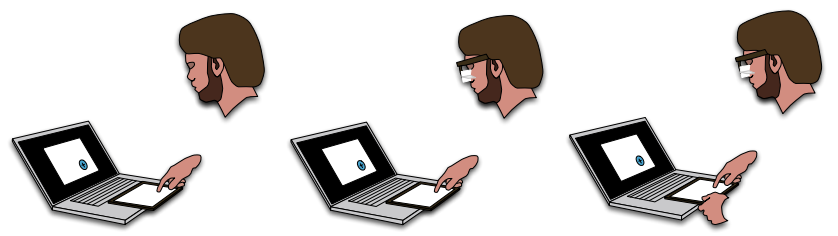

Figure 4: 1HAND, 1HANDBLINDERS and 2HANDSBLINDERS.

were not allowed to look at it and had to wear the blinder glasses shown on Figure 1. In 1HAND and 1HANDBLinders, participants were not allowed to use their non-dominant hand which had to stay away from the desk and the input device. In 2HANDSBLINDERS, they were asked to hold the input device with it in place, without moving. As Gustafson et al. had shown that the ability to see the hands outperforms the tactile cues they provide [7], we did not consider having a fourth condition with both hands allowed and visible.

To summarize, participants could always see the laptop's display and use their dominant hand's index finger to point at locations on the input surface. Their non-dominant hand was used only in 2HANDSBLINDERS where it held in place the input device, and they could look at the input surface only in 1HAND.

The presentation order of InPUT Condition and DeviCE SIZE was counterbalanced across participants using a balanced Latin Square design. TARGET SIZE was presented in descending order. Each Block consisted of 3 repetitions of the 9 TARGET POSITION presented in a pseudo-random order. The experimental design was thus: 12 participants $\times 3$ INPUT CONDITION $\times 2$ DEVICE SIZE $\times 3$ BLOCK $\times 3$ TARGET SIZE $\times 9$ TARGET POSITION $\times 3$ Repetitions $=17,496$ total trials.

For each trial, we recorded the first touch position of each attempt and the number of attempts to select the targets. The experiment lasted around 50 minutes for each participant.

\section{RESULTS}

The dependent variables were the success rate, the number of failed attempts and the targeting error.

\section{Success rate and number of failed attempts}

Targets that were not selected on first attempt were marked as errors. In what follows, the success rate is the percentage of targets successfully selected on first attempt. The mean number of failed attempts is the average number of times the participant attempted to select the target and failed (5 at most). In the LARGE condition for example, as illustrated in Figure 5, the success rate is $54 \%$ and the mean number of failed attempts

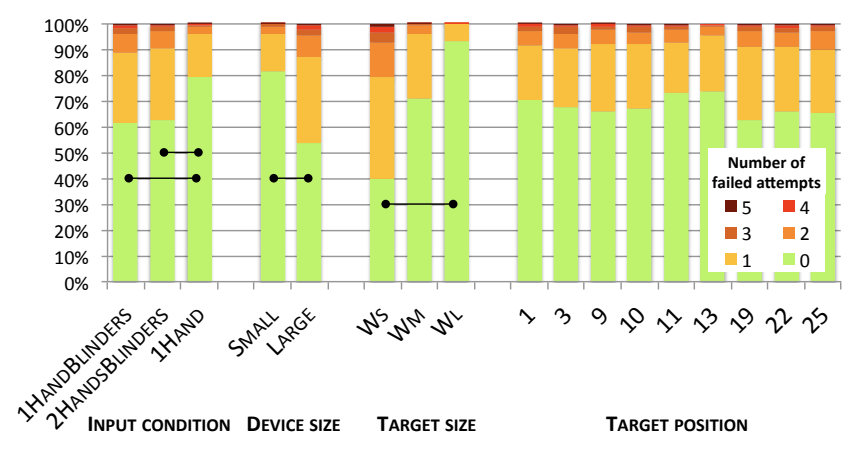

Figure 5: Number of failed attempts across INPUT CONDITION, DEVICE SIZE, TARGET SIZE and TARGET POSITION (from left to right). Connections between bars represent statistically significant differences.

is $0.66(0.54 \times 0+0.33 \times 1+0.08 \times 2+0.03 \times 3+0.01 \times 4+$ $0.01 \times 5)$.

A Friedman analysis showed a significant main effect of BLOCK $\left(\chi^{2}=13.2, \mathrm{df}=2, \mathrm{p}=0.001\right)$ on the success rate and the mean number of failed attempts $\left(\chi^{2}=10.2, \mathrm{df}=2, \mathrm{p}<0.01\right)$. Pairwise comparisons ${ }^{1}$ showed significant differences between the first block and the two subsequent ones for the success rate $(\mathrm{p}<0.05)$, and between the first block and the second one for the mean number of failed attempts $(\mathrm{p}<0.05)$, showing a learning effect. The first block was thus removed from subsequent analysis. We also considered trial positions at least three standard deviations away from the mean position for each condition as outliers and removed them from the data analysis $(0.9 \%$ of the trials).

The Friedman analysis showed significant main effects of INPUt CONDITION $\left(\chi^{2}=18.7, \mathrm{df}=2, \mathrm{p}<0.001\right)$, Device Size $\left(\chi^{2}=12, \mathrm{df}=1\right.$, $\mathrm{p}<0.001)$, TARGET SIZE $\left(\chi^{2}=24, \mathrm{df}=2, \mathrm{p}<0.001\right)$ and TARget posiTION $\left(\chi^{2}=30.4, \mathrm{df}=8, \mathrm{p}<0.001\right)$ on the number of failed attempts. Pairwise comparison showed significant differences between 1 HAND and the other input conditions ( $p<0.01,1$ HAND: 0.26 , 1HANDBLINDERS: 0.56 , 2HANDSBlinders: 0.51 ). The mean number of failed attempts fell from 0.66 with LARGE to 0.23 with SMALL. Significant differences $(\mathrm{p}<0.001)$ were found between Ws (0.93) and WL (0.07). Post-hoc analysis for TARGET POSITION did not reveal any significant difference in spite of the main effect.

The number of failed attempts for each condition is represented on Figure 5. Overall participants were more successful when they could see the input device, used the smaller input surface or selected larger targets.

\section{Targeting error}

Targeting error is computed for all trials, whether succeeded or not, as the distance (in $\mathrm{mm}$ ) between the location of the first attempt and the target center. A low targeting error corresponds to a high accuracy. Friedman analysis showed a significant effect of ВLOCK $\left(\chi^{2}=15.2, \mathrm{df}=2, \mathrm{p}<0.001\right)$ on targeting error and pairwise comparisons showed a significant difference between the first block and the following ones $(p<0.01)$. As a result the first block was removed from subsequent analysis.

\footnotetext{
${ }^{1}$ Post-hoc analysis were adjusted using Bonferroni correction.
} 
As the targeting error did not follow a normal distribution, we used the "Aligned Rank Transform" [22] and ran a repeated measures ANOVA on the aligned ranks to investigate possible interactions between the factors.

The analysis showed a significant main effect of DevicE SIZE $\left(F_{1,11}=489, \mathrm{p}<0.001\right)$ and INPUT CONDITION $\left(F_{2,22}=72.1, \mathrm{p}<0.001\right)$, and a significant interaction of DEvice SIZE $\times$ INPUT CONDITION $\left(F_{2,22}=28.4, \mathrm{p}<0.001\right)$ on targeting error. Mean targeting error for SMALL and LARGE were respectively 5.9 and $11.1 \mathrm{~mm}$. We hypothesize the lower targeting error obtained in the SMALL condition could be explained by the smaller displacements to perform on the small device: participants could perform small movements more precisely than large ones.

Pairwise comparisons showed significant differences between all levels of InPUT CONDITION (1 HandBLINDERS: $9.8 \mathrm{~mm}$, 2HANDSBLINDERS: $9.4 \mathrm{~mm}, 1$ HAND: $6.2 \mathrm{~mm}$; $<<0.001$ ). Looking at the input surface clearly reduced targeting error, even though nothing was displayed there. Overall, 2HANDSBlinders reduced targeting error by $4 \%$ compared to 1 HANDBLINDERS, and 1 HAND reduced it by $37 \%$. But the significant interaction of DEVICE SIZE $\times$ INPUT CONDITION revealed there was no significant difference between 2HANDSBLINDERS and 1HANDBLINDERS for SmALL, while there was a significant difference between these two conditions in the LARGE condition (Figure 6).

We found a significant main effect of TARGET SIZE $\left(F_{2,22}=12.7\right.$, $\mathrm{p}<0.001)$ and a significant interaction of TARGET SIZE $\times$ DEVICE SIZE $\left(F_{2,22}=7.0, \mathrm{p}=0.005\right)$. Post-hoc analysis showed that targeting error is significantly $(\mathrm{p}<0.001)$ higher for $\mathrm{WL}_{\mathrm{L}}(9.1 \mathrm{~mm})$ compared to $\mathrm{WM}_{\mathrm{M}}(8.2 \mathrm{~mm})$ and $\mathrm{Ws}(8.0 \mathrm{~mm})$. Participants may have been less careful with large targets, which may have seem easier to select. Pairwise comparisons also revealed that targeting error stopped decreasing for the LARGE condition below target size WM: no significant difference was found between Ws and WM for LARGE while significant differences were found between these two sizes for the Small condition. No significant interaction was found for TARGET SIZE $\times$ INPUT CONDITION.

We found a significant main effect of TARGET POSITION $\left(F_{8,88}=12.25, \mathrm{p}<0.001\right)$ and a significant interaction for TARGET POSITION $\times$ INPUT CONDITION $\left(F_{16,176}=5.39, \mathrm{p}<0.001\right)$ on targeting error. Targeting error was minimum for the target located at the center, followed by targets in the upper left quarter and then targets on the diagonal (targets 9, 10 and 22). Most participants let their fingers centered above the device which required them to fold the fingers to reach targets at the bottom right corner. For these targets (19 and 25), the targeting error was the highest. Post-hoc analysis revealed that targeting error for target 25 is significantly lower in the 1HAND condition. However for targets 9 and 19 targeting error is significantly higher in the 1HAND condition, probably because these targets are neither positioned on borders nor at the center so they do not benefit from visual guidances to estimate their position.

\section{Minimum target size in motor space}

Based on our experimental data, we can derive the minimal target size in motor space participants can select on first attempt with a $95 \%$ probability. We examined the dispersion of all touches around each target. We found the average touch
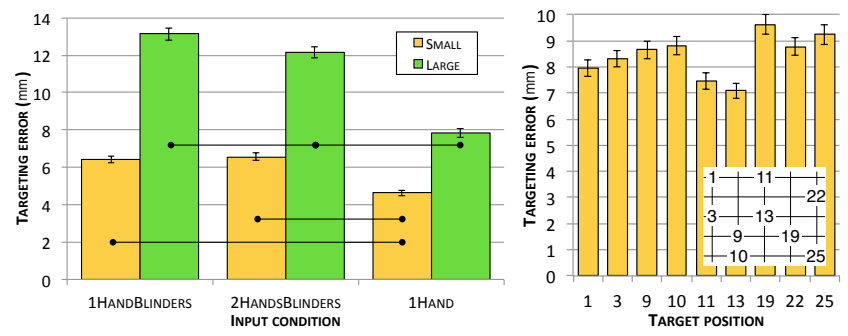

Figure 6: Targeting error for DEVICE SIZE and INPUT CONDITION (left), and TARGET POSITION (right). Connections between bars represent statistically significant differences.

position is offset $0.4 \mathrm{~mm}$ right and $0.8 \mathrm{~mm}$ top from the target center.

A repeated-measures MANOVA on the first touch position showed a significant main effect of INPUT CONDITION $\left(F_{4,8}=5.8\right.$, $\mathrm{p}=0.019)$ and Device SIZE $\left(F_{2,10}=9.1, \mathrm{p}<0.01\right)$ on the smallest target size. The corresponding minimal target sizes are reported in Table 1 . These results show that the ability to look at the device results in higher precision than holding it with the nondominant hand. It also confirms that participants were almost two times more precise on the small device $(82 \%)$.

\begin{tabular}{lcccc}
\hline \multicolumn{2}{l}{ INPUT CONDITION } & $\begin{array}{c}\text { 1HAND } \\
\text { BLINDERS }\end{array}$ & $\begin{array}{c}\text { 2HAND } \\
\text { BLINDERS }\end{array}$ & 1HAND \\
\hline \multirow{2}{*}{ DEVICE SIZE } & SMALL & 22.3 & 23.2 & 16.8 \\
\cline { 2 - 5 } & LARGE & 45.2 & 41.3 & 27.8 \\
\hline
\end{tabular}

Table 1: Minimum target size (in $\mathrm{mm}$ ) to acquire a target on first attempt with $95 \%$ of chance, across INPUT CONDITION and DEVICE SIZE.

\section{Spatial distribution of the first touch position}

Figure 7 shows all trials split by DEvice SIZE and INPUT CONDITION. Ovals represent the bivariate normal distribution of first touch positions for each TARGET POSITION, represented by cross-hairs.

Across all conditions, touches tend to be shifted towards the center of the device even though the presentation order of target positions was randomized. This can be explained by the dominant strategy which consisted in keeping the fingers around the center of the device. In addition to this deviation, the distribution of the 1HAND condition is moved towards the bottom right in comparison with the two other input conditions. The center of the ellipse for 1HAND is generally on the bottom right side of the other centers.

Vogel, Baudisch and Holz [19, 9] have shown that most users assume the contact point considered by the machine to be located above the nail center, or at its extremity. Yet touch interfaces use the centroid of contact shapes which is shifted towards the south-west for right fingers. This offset between what people consider doing and what the computer interprets introduces a misunderstanding of the perceived input point model. In our experiment, when participants were allowed to look at the device, we assume they mentally projected targets and put their finger at the corresponding locations. The specific offset observed in this condition can be explained by the above perceived input model. As all participants were right-handed, their finger was oriented towards the top left 
corner, and the offset is in the bottom right direction. The two blinded conditions (1HANDBLINDERS and 2HANDSBLINDERS) are not concerned by this deviation. Participants could not see their finger and the input model they built is only based on the on-screen mark's position.
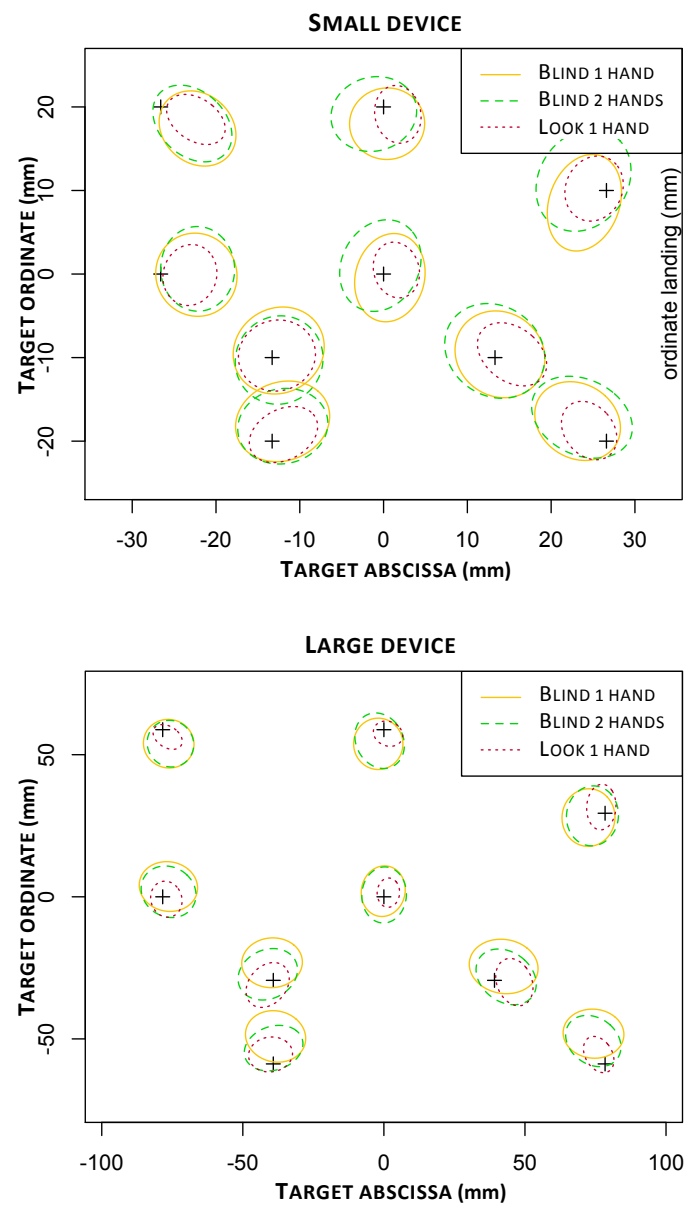

Figure 7: Bivariate standard-deviation across DEVICE SIZE and INPUT CONDITION. Cross-hairs represent each target center position in device space. Ellipses gather $68 \%$ of touch points for each position.

To summarize, the main finding of this first experiment is that targeting error is not constant across different input device sizes: it was smaller with the small device. We have also shown that looking at the input device helps acquiring targets while using the non dominant hand as a reference frame does not help significantly. The position of targets also matters with the ones positioned on the dominant hand side being the most difficult to acquire while targets at the center and corners being easier to select. This experiment did not examine the effect of the scale at which targets are displayed, nor the difference of aspect ratio between the input surface and the display. These factors are examined in the next experiment.

\section{EXPERIMENT 2: SCALE EFFECTS AND ASPECT RATIO}

The purpose of this second experiment was to evaluate the influence of form factors of the display on absolute indirecttouch pointing performance. We hypothesized that the display scale, i.e. the size of targets relative to that of the workspace, would have no impact on performance. We also hypothesized that similar input and output aspect ratios would lead to better performance.

\section{Apparatus, task and procedure}

To evaluate a larger range of display sizes and aspect ratios, we replaced the display used in Experiment 1 by a 50" monitor $(1106 \times 622 \mathrm{~mm}, 1365 \times 768$ pixels $)$. Part of the display was masked to simulate the different display sizes and aspect ratios while keeping the same pixel density across conditions As we wanted to evaluate a condition with a display surface smaller than the input one, we decided to use the iPad (the LARGE device) rather than the iPod Touch. The experiment ran on a PC (Windows 7, $2.7 \mathrm{GHz}, 4 \mathrm{~GB}$ of RAM). The monitor was positioned vertically $90 \mathrm{~cm}$ away from participants. The input device was positioned horizontally on a desk, 60 $\mathrm{cm}$ away from participants' eyes.

The task and procedure were the same as in Experiment 1 except that the size and aspect ratio of the on-screen workspace varied accross conditions. We used the 1HAND input condition, i.e. participants could but were not enforced to look at the input device, and they were not allowed to use their nondominant hand. Targets were always presented as disks on the display but stretched on the input surface to accomodate the possible differences in aspect ratios. As in Experiment 1, targets were never shown on the input device.

\section{Participants}

12 unpaid volunteers who did not participate in Experiment 1 participated in this one: 5 female and 7 male, all right-handed, with a mean age of $31.8(\mathrm{SD}=8.4)$.

\section{Design}

A repeated measures within-subjects design was used. The independent variables were the height of the workspace (Workspace height), its aspect ratio (Aspect RATIo), the target size (TARGET SIZE) and its position (TARGET POSITION).

WORKSPACE HEIGHT was evaluated with 3 levels $(\mathrm{Hs}=74 \mathrm{~mm}$, two times smaller than the iPad; $\mathrm{Hm}=147 \mathrm{~mm}$, like the iPad; $\mathrm{HL}=294 \mathrm{~mm}$, two times bigger than the $\mathrm{iPad})$.

ASPECT RATIO was evaluated with 3 levels $(\mathrm{RM}=4: 3, \mathrm{RL}=16: 9$ and $\mathrm{RXL}=32: 10$, the corresponding workspace sizes being presented in Table 2). A ratio of 32:10 corresponds to two modern widescreen computer monitors (16:10) side by side. A ratio of $16: 9$ is typical of modern widescreen TVs. Although somewhat less popular today, $4: 3$ is the ratio of the input device (the iPad) and supports comparison with Experiment 1.

\begin{tabular}{ccccc}
\hline \multicolumn{2}{c}{ WORKSPACE HEIGHT } & HS & HM & HL \\
\hline \multirow{3}{*}{ ASPECT RATIO } & RM & $98 \times 74$ & $196 \times 147$ & $392 \times 294$ \\
\cline { 2 - 5 } & RL & $131 \times 74$ & $261 \times 147$ & $523 \times 294$ \\
\cline { 2 - 5 } & RXL & $235 \times 74$ & $470 \times 147$ & $941 \times 294$ \\
\hline
\end{tabular}

Table 2: Workspace dimensions (in $\mathrm{mm}$ ) depending on WORKSPACE HEIGHT and ASPECT RATIO.

TARgET SIZE was evaluated with 2 levels $(\mathrm{WM}=20 \mathrm{~mm}$ and $\mathrm{WL}_{\mathrm{L}}=40 \mathrm{~mm}$ ). As Experiment 1 did not show any significant 


\begin{tabular}{|c|c|c|c|c|c|}
\hline \multirow[t]{4}{*}{ WM } & \multicolumn{2}{|c|}{ WORKSPACE HEIGHT } & Hs & НM & HL \\
\hline & \multirow{3}{*}{ ASPECT RATIO } & $\mathrm{RM}$ & $20 \times 20$ & $10 \times 10$ & $5 \times 5$ \\
\hline & & RL & $15 \times 20$ & $17.5 \times 10$ & $3.7 \times 5$ \\
\hline & & $\mathrm{RXL}$ & $8.3 \times 20$ & $4.2 \times 10$ & $2.1 \times 5$ \\
\hline \multirow[t]{4}{*}{ WL } & \multicolumn{2}{|c|}{ WORKSPACE HEIGHT } & $\mathrm{Hs}$ & HM & $\mathrm{HL}$ \\
\hline & \multirow{3}{*}{ ASPECT RATIO } & RM & $40 \times 40$ & $20 \times 20$ & $10 \times 10$ \\
\hline & & RL & $30 \times 40$ & $15 \times 20$ & $7.5 \times 10$ \\
\hline & & $\mathrm{RXL}$ & $16.7 \times 40$ & $8.3 \times 20$ & $4.2 \times 10$ \\
\hline
\end{tabular}

Table 3: Target size in motor space (in $\mathrm{mm}$ ) corresponding to the displayed TARGET SIZE depending on the WORKSPACE HEIGHT and ASPECT RATIO. Highlighted cells are the ones for which different visual conditions lead to the same target size in motor space.

difference between Ws and WM on the LARGE device, we removed Ws to shorten the duration of the experiment. In the (Ws, RxL, HL) condition, on-screen targets would have been mapped to $1.05 \mathrm{~mm}$ targets in motor space, a size most probably too small to be selected anyway. Table 3 shows the target sizes in motor space corresponding to $\mathrm{WM}_{\mathrm{M}}$ and $\mathrm{WL}_{\mathrm{L}}$ for the different combinations of ASPECT RATIO and WORKSPACE HEIGHT.

As the effect of TARGET POSITION had already been evaluated with 9 levels in Experiment 1, we decided to evaluate it with fewer levels this time. We chose 4 positions with contrasted effects: the easiest target at the center (13), the top left corner position also easy to acquire (1), a more difficult one on the diagonal (22), and a difficult one most probably covered by the hand (19).

The presentation order of WORKSPACE HEIGHT and ASPECT RATIO was counterbalanced across participants following a balanced Latin Square. TARGET SIZE was presented in descending order. Each BLOCK consisted of 3 repetitions of the 4 TARGET POSITION presented in a pseudo-random order. The experimental design was thus: 12 participants $\times 3$ WORKSPACE HEIGHT $\times 3$ ASPECT Ratio $\times 3$ Block $\times 2$ TARget Size $\times 4$ TARget Position $\times 3$ Repetitions $=7,776$ total trials. For each trial we recorded the first touch position of each attempt and the number of attempts to select the target. The experiment lasted around 20 minutes for each participant.

\section{RESULTS}

The dependent variables were again the success rate, the number of failed attempts and the targeting error, as previously defined. We considered trial positions at least three standard deviations away from the mean position as outliers and removed them from the data analysis ( $0.8 \%$ of the trials).

\section{Success rate and number of failed attempts}

The number of failed attempts for each condition is represented on Figure 8. A Friedman analysis did not show a significant main effect of BLOCK neither on success rate $\left(\chi^{2}=4.04\right.$, $\mathrm{df}=2, \mathrm{~ns})$ nor on the mean number of failed attempts $\left(\chi^{2}=3.43\right.$, $\mathrm{df}=2, \mathrm{~ns})$. But the analysis showed significant main effects of Workspace height $\left(\chi^{2}=24, \mathrm{df}=2, \mathrm{p}<0.001\right)$, Aspect Ratio $\left(\chi^{2}=22.2\right.$, $\mathrm{df}=2, \mathrm{p}<0.001)$, TARGET SizE $\left(\chi^{2}=12, \mathrm{df}=1, \mathrm{p}<0.001\right)$ and TARgeT POSITION $\left(\chi^{2}=24.2, \mathrm{df}=3, \mathrm{p}<0.001\right)$ on the number of failed attempts. Pairwise comparisons revealed significant differences for it between Hs and HL for WorKsPaCE HEIGHT (0.10 and 1.04, $\mathrm{p}<0.001)$ and between RM and RXL for ASPECT RATIO ( 0.30 and $0.80, \mathrm{p}<0.001)$. For TARGET POSITION, we found significant differences between targets 13 and $19(0.32$ and $0.66, \mathrm{p}<0.05)$, and between 13 and $22(0.32$ and $0.53, \mathrm{p}<0.05)$.

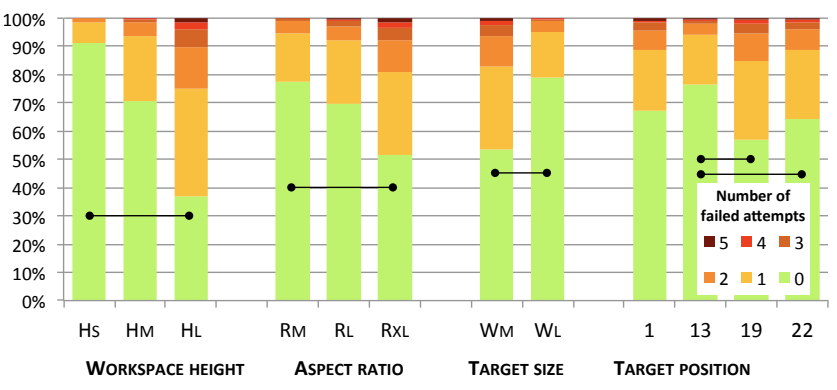

Figure 8: Number of failed attempts across WORKSPACE HEIGHT, AsPECT RATIO, TARGET SIZE and TARGET POSITION (from left to right). Connections between bars represent statistically significant differences.

\section{Targeting error}

A Friedman analysis showed a significant main effect of BLOCK $\left(\chi^{2}=8.17, \mathrm{df}=2, \mathrm{p}=0.017\right)$ on targeting error, but post-hoc analysis did not reveal any significant difference between the blocks. As a result the three blocks were conserved for subsequent analysis.

A repeated measures ANOVA showed a significant main effect of WORKSPACE HEIGHT $\left(F_{2,22}=7.06, \mathrm{p}<0.01\right)$ and ASPECT RATIO $\left(F_{2,22}=11.72, \mathrm{p}=<0.001\right)$ on targeting error, but no significant WORKSPACE HEIGHT $\times$ ASPECT RATIO interaction. Post-hoc analysis showed significant differences between Hs and the other heights Hм and HL $(10.2 \mathrm{~mm}, 8.9 \mathrm{~mm}$ and $8.7 \mathrm{~mm}, \mathrm{p}<0.001$, Figure 9). The larger workspaces resulted in smaller targets in motor space. The lower targeting error observed for $\mathrm{HM}$ and $\mathrm{HL}$ might result from increased participant attention in response to their lower success rate (Figure 8). Post-hoc comparisons showed significant differences between RxL and the two other aspect ratios RM and RL $(9.8 \mathrm{~mm}, 8.9 \mathrm{~mm}$ and $9.0 \mathrm{~mm}, \mathrm{p}<0.01)$. Targeting error increased as the aspect ratio increased: the horizontal stretching impaired the correct estimation of target position.

The analysis showed a significant main effect of TARGET SIZE $\left(F_{1,11}=25.83, \mathrm{p}<0.001\right.$, Figure 9). As in Experiment 1, smaller targets led to reduced targeting error (WL: $9.6 \mathrm{~mm}$, WM: $9.0 \mathrm{~mm}$ ). We again hypothesize that participants may have been less careful with large targets which appear easier to select.

A significant interaction was found between ASPECT RATIO and TARGET SIZE $\left(F_{2,22}=6.30, \mathrm{p}<0.01\right)$ but post-hoc comparisons did not reveal any significant difference. Targeting error decreased when the target size decreased in motor space, and as the workspace and input aspect ratios got closer. It appears to reach a floor for the smallest target size (WM) with the aspect ratios RL and RM $(8.5 \mathrm{~mm}$ and $8.7 \mathrm{~mm})$. Participants could not further reduce the error when the aspect ratio became smaller (from RL to RM). 
We also found a significant main effect of TARGET POSITION $\left(F_{3,33}=34.73, \mathrm{p}<0.001\right)$ and a significant interaction between TARGet position and Aspect Ratio $\left(F_{6,66}=4.44, \mathrm{p}<0.001\right)$. Post-hoc comparisons showed significant differences between all target positions $(p<0.001)$ in the same order as in Experiment 1 (Figure 9). Post-hoc analysis revealed that the significant interaction comes from targets 13 (the center) and 22 (on the right border). For 13, participants were significantly more precise in RXL compared to the other aspect ratios (RM: 7.3 $\mathrm{mm}$, RL: $7.3 \mathrm{~mm}$, RXL: $6.6 \mathrm{~mm}, \mathrm{p}<0.05)$. In contrast, participants were significantly less precise for 22 as the aspect ratio got wider (RM: $9.4 \mathrm{~mm}$, RL: $9.3 \mathrm{~mm}$, RxL: $11.3 \mathrm{~mm}, \mathrm{p}<0.05$, Figure 10 ). Although not significant, targets 1 and 19 also led to higher targeting error for the larger aspect ratio. The center of the workspace remains a position easier to reach even when targets are stretched in motor space.

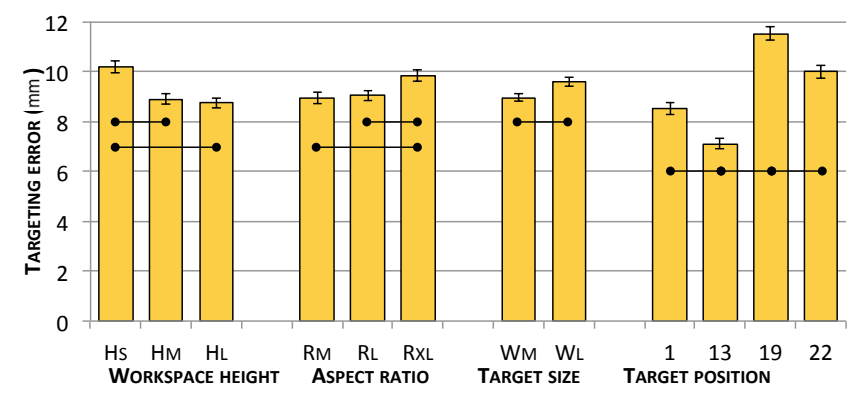

Figure 9: Targeting error (in mm) for WORKSPACE HEIGHT, ASPECT ratio, Target Size and TARget position. Connections between bars represent statistically significant differences.

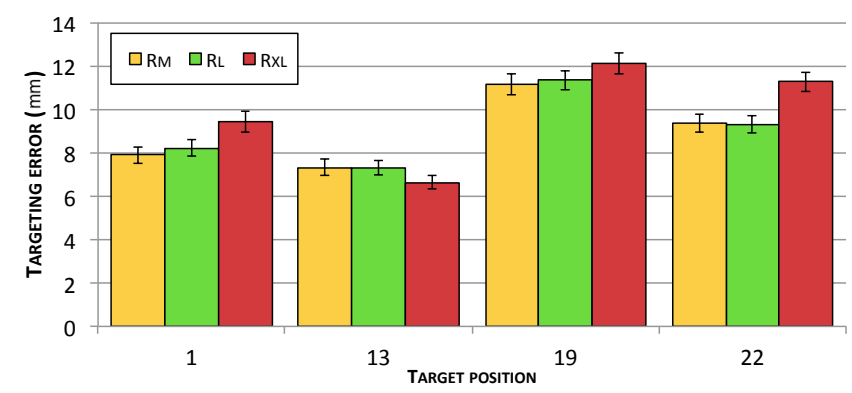

Figure 10: Targeting error (in mm) for TARGET POSITION and ASPECT RATIO.

\section{Minimum target size in motor space}

Minimum target sizes containing 95\% of first touch positions across WORKSPACE HEIGHT and ASPECT RATIO are presented in Table 4. The size obtained for the Rм, Hм condition $(30.2 \mathrm{~mm})$ is consistent with the one obtained for the LARGE,1 HAND condition of Experiment 1 (27.8 mm, see Table 4). Overall, the average touch position is offset towards the bottom-right direction of the target center (1.5 $\mathrm{mm}$ right, $1.1 \mathrm{~mm}$ bottom).

A repeated measures MANOVA on the first touch position showed a significant interaction between WORKSPACE HEIGHT and Aspect Ratio $\left(F_{8,4}=8.1, \mathrm{p}<0.05\right)$. Posthoc comparisons revealed the interaction is due to Hs for which no significant difference was found across the aspect ratios while significant differences were observed for the other workspace heights.

\begin{tabular}{lcccc}
\hline WORKSPACE HEIGHT & $\mathrm{HS}$ & $\mathrm{HM}$ & $\mathrm{HL}$ \\
\hline \multirow{2}{*}{ ASPECT RATIO } & $\mathrm{RM}$ & 35.5 & 30.2 & 28.2 \\
\cline { 2 - 5 } & $\mathrm{RL}$ & 34.5 & 30.7 & 30.3 \\
\cline { 2 - 5 } & $\mathrm{RXL}$ & 37.6 & 32.1 & 35.1 \\
\hline
\end{tabular}

Table 4: Minimum target size in $\mathrm{mm}$ (contains $95 \%$ of first touch positions) across WORKSPACE HEIGHT and ASPECT RATIO.

Target sizes in motor space were largest for Hs and it appears their larger size made them easier to select in spite of their stretching in motor space. As a result it is most likely that participants did not reach their limit in minimum target size for that workspace height.

The effect of the visual scale on precision can be analyzed for conditions giving the same target size in motor space. Нм, WM and $\mathrm{HL}_{\mathrm{L}} \mathrm{WL}$ produce identical target sizes in motor space (10 $\mathrm{mm}$ according to Table 3 ) but the corresponding displayed targets are two times bigger in the second condition. The resulting minimum target sizes shows a difference equal to $1.3 \mathrm{~mm}$ ( 29.9 vs. $31.2 \mathrm{~mm}$ ), suggesting that visual scale has little influence on precision.

\section{Spatial distribution of first touch position}

Figure 11 shows all trials split by Aspect Ratio. Ovals represent the bivariate normal distribution of first touch position for each TARGET POSITION represented by cross-hairs. The center target shows an homogeneous distribution compared to the other ones and the corresponding accuracy remains steady across all ASPECT RATIO, confirming this target is easier to select regardless of the aspect ratio. For the other positions it is interesting to notice how the ellipses get shifted across the different aspect ratios. In particular the ellipses for RxL show the highest deviation, confirming the higher difficulty to point at target centers when they are stretched in motor space.

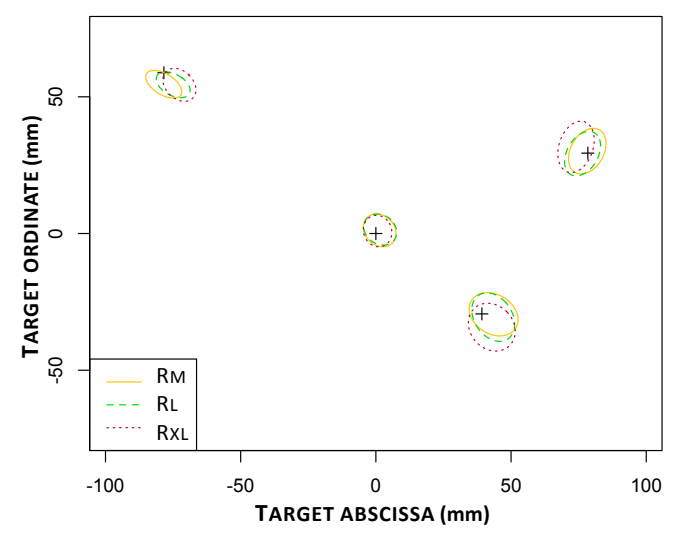

Figure 11: Bivariate standard-deviation across WORKSPACE HEIGHT. Cross-hairs represent each target center position in device space. Ellipses gather $68 \%$ of touch points for each position.

\section{DISCUSSION}

We ran two experiments to investigate the factors influencing performance in absolute indirect-touch pointing tasks. We hereafter provide guidelines for the design of such tasks but also discuss the limits of our experiments. Finally, we 
present new interaction scenarios based on our design guidelines where absolute indirect-touch pointing could be useful.

Experiment 1 shows that being able to look at the input surface improves performance compared to blinders conditions. When participants relied solely on proprioception, they were moderately but significantly less efficient (about 20\% less for success rate, and $3 \mathrm{~mm}$ more for targeting error). In this experiment, we could not strictly control or measure how often participants looked at the input surface, but we can hypothesize that seeing it at least from time to time facilitates finger positioning, even if nothing is displayed on it.

Recommendation 1: Even if not a display, users should be able to see the input surface in absolute indirect-touch pointing tasks. As a consequence, the boundaries of the surface should be clearly distinguishable.

Target position also influences success rate and accuracy. Targets near the center or in the corners of the input surface are easier to select. For right-handed people, targets in the NorthWest quadrant of the workspace are also relatively easy to select. The North-East and South-West quadrants are equally difficult. The most difficult targets are those in the South-East quadrant which require to fold the finger.

Recommendation 2: Designers should take the handedness of the users into account for all absolute indirect-touch pointing tasks. When possible, frequently accessed objects should be put in the middle, in the corners or in the easier quadrant of the workspace (NW for right-handed people). The difficult quadrants (NE \& SW) could be used for less frequent targets. The most difficult one (SE) could be used for irreversible actions, for example.

Experiment 2 shows that success rate and targeting error are not affected by the scale at which targets are represented onscreen: for a given size in motor space, a target displayed two times bigger is not easier or more difficult to select. The first experiment shows that the distribution of touches is shifted towards the center of the input surface. In Experiment 2 , the shift gets more pronounced as the aspect ratio of the workspace diverged from that of the input surface.

Recommendation 3: In absolute indirect-touch situations, the display scale does not matter, but input and output aspect ratios do. To increase the success rate and reduce the targeting error of pointing tasks, designers should use similar ratios for the input surface and the on-screen workspace.

Our participants were more precise with smaller targets and $16.8 \mathrm{~mm}$, about the width of a finger, was the limit in size they could reliably acquire on first attempt when they could see the input surface (Table 1). This value is larger than the $10.5 \mathrm{~mm}$ found by Vogel and Baudisch using an absolute direct mapping on a PDA [19]. The co-localization of the input and display surfaces probably explains the higher accuracy they observed. Our value is closer to the $15 \mathrm{~mm}$ found by Holz and Baudisch (with a direct mapping) on the iGesture touchpad [9] and the $17.7 \mathrm{~mm}$ found by Gustafson et al. on the palm [6]. The limit we found when participants could not see the input surface was about $23 \mathrm{~mm}$ (Table 1), a value consistent with those found in touch typing studies [18, 3].

Most importantly, we have shown that the size of the input surface noticeably affects success rate and accuracy. In Experiment 1 , accuracy was higher on the small input surface (Table 1). The ability to acquire small targets decreases if the size of the input surface increases, which seems counterintuitive. We hypothesize the lower accuracy observed with the large input surface is due to the larger distances to cover in motor space. In absence of visual feedback during the task, the movement to execute can be considered a ballistic one. And due to noise in the motor system, the endpoint of a ballistic movement may not exactly occur at the anticipated location. Lin et al. have actually shown that the endpoint error of a ballistic movement is linearly related to the movement amplitude [11]. We can thus assume that the targeting accuracy is linearly related to the size of the input surface.

Further experiments and analysis are required to validate this hypothesis and understand the exact relation between the size of the input surface and the minimum target size in motor space. This could have important implications since the minimum target size in motor space has a direct impact on the size of on-screen objects: to reliably acquire targets on first attempt, their on-screen size should be larger than the minimum size in motor space times the ratio of the diagonals of the workspace and the input surface.

Recommendation 4: Designers of absolute indirect-touch interfaces should pay attention to the minimum target size in motor space and use it to check whether the on-screen interactors can be reliably acquired. Researchers should further investigate the nature of absolute indirect-touch pointing tasks and the effect of larger movements on accuracy.

To conclude, we would like to propose a few examples in which the results of our two studies might be useful.

Let us consider the owner of a laptop with a 15" $(323 \times$ $202 \mathrm{~mm}$ ) monitor and a $105 \times 76 \mathrm{~mm}$ touchpad who would want to use it for absolute pointing. In this configuration, a minimum target size in motor space of $21 \mathrm{~mm}$ (extrapolated from our results) leads to a minimum size for on-screen objects of $62 \mathrm{~mm}$. The form factors of current touchpads do not favor absolute interaction. Nevertheless, considering the size of the objects typically displayed on a laptop screen, a reasonable use would be for managing windows, for example. The user could enter a special mode, by pressing a key for example. While in this mode, she would be able to select and move windows with absolute taps and drag gestures on the touchpad. Using this absolute mode instead of the traditional relative one, she would be able to manipulate several windows at the same time.

The same person might have access to a wall display made of multiple similar screens. In this situation, she could use absolute indirect-touch pointing to indicate where she wants to send a window displayed on her laptop. She could enter a local absolute mode, designate the window on her screen by tapping at the corresponding location on the touchpad, then switch to a remote absolute mode mapping the wall display 
onto her touchpad and tap at the appropriate location. Considering the $21 \mathrm{~mm}$ minimum size in motor space, the touchpad could be divided in 5 horizontally and 4 vertically and could thus address $5 \times 4$ screens.

A last scenario would be using a wearable multi-touch touchpad on the arm to interact with a wristwatch presenting a high resolution display. Considering a $30 \times 30 \mathrm{~mm}$ screen and a $70 \times 70 \mathrm{~mm}$ touchpad, then the smallest target size in motor size would be equal to $18 \mathrm{~mm}$ and the corresponding smallest target size on screen would be equal to $8 \mathrm{~mm}$.

\section{CONCLUSION}

Considering the lack of knowledge regarding the form factors and input conditions affecting performance on absolute indirect-touch pointing tasks, we ran two experiments to systematically investigate these factors. The first experiment focused on input device size and input conditions and revealed that users get higher performance when they can look at the input surface (even if nothing is displayed on it). In addition we found that the smallest target size users can acquire in motor space is not constant across different input dimensions but degrades as the input size increases. The second experiment focused on scale effects and aspect ratio and revealed users' performance is not affected by scale but that aspect ratio matters: similar input and output aspect ratios lead to better performance. This findings led us to list four main recommendations for the design of touch input surfaces with applications supporting absolute direct interaction. Our results suggest the minimum target size in motor space linearly increases with the size of the input surface. As future work we plan to investigate in a systematic way the influence of input surface dimensions on this minimum target size.

\section{ACKNOWLEDGMENTS}

This work was supported by ANR (InSTInCT, ANR-09CORD-013) and the Conseil Régional Nord - Pas de Calais.

\section{REFERENCES}

1. Benko, H., Izadi, S., Wilson, A. D., Cao, X., Rosenfeld, D., and Hinckley, K. Design and evaluation of interaction models for multi-touch mice. In Proc. GI' 10 (2010), 253-260.

2. Casiez, G., and Roussel, N. No more bricolage! Methods and tools to characterize, replicate and compare pointing transfer functions. In Proc. UIST' 11 (2011), 603-614.

3. Findlater, L., Wobbrock, J. O., and Wigdor, D. Typing on flat glass: examining ten-finger expert typing patterns on touch surfaces. In Proc. CHI'11 (2011), 2453-2462.

4. Forlines, C., Vogel, D., and Balakrishnan, R. Hybridpointing: fluid switching between absolute and relative pointing with a direct input device. In Proc. UIST '06, ACM (2006), 211-220.

5. Forlines, C., Wigdor, D., Shen, C., and Balakrishnan, R. Direct-touch vs. mouse input for tabletop displays. In Proc. CHI '07 (2007), 647-656.

6. Gustafson, S., Holz, C., and Baudisch, P. Imaginary phone: learning imaginary interfaces by transferring spatial memory from a familiar device. In Proc. UIST'11 (2011), 283-292.
7. Gustafson, S. G., Rabe, B., and Baudisch, P. M. Understanding palm-based imaginary interfaces: the role of visual and tactile cues when browsing. In Proc. CHI'13 (2013), 889-898.

8. Hall, A. D., Cunningham, J. B., Roache, R. P., and Cox, J. W. Factors affecting performance using touch-entry systems: Tactual recognition fields and system accuracy. Journal of Applied Psychology 73, 4 (1988), 711-720.

9. Holz, C., and Baudisch, P. The generalized perceived input point model and how to double touch accuracy by extracting fingerprints. In Proc. CHI' 10 (2010), 581-590.

10. Hutchins, E. L., Hollan, J. D., and Norman, D. A. Direct manipulation interfaces. HCI 1, 4 (Dec. 1985), 311-338.

11. Lin, R. F., and Drury, C. G. Verification of models for ballistic movement time and endpoint variability. Ergonomics 56, 4 (2013), 623-636.

12. Lin, S.-Y., Su, C.-H., Cheng, K.-Y., Liang, R.-H., Kuo, T.-H., and Chen, B.-Y. PUB (point upon body): exploring eyes-free interaction and methods on an arm. In Proc. UIST'11 (2011), 481-488.

13. Malik, S., Ranjan, A., and Balakrishnan, R. Interacting with large displays from a distance with vision-tracked multi-finger gestural input. In Proc. UIST '05 (2005), 43-52.

14. McCallum, D. C., and Irani, P. ARC-Pad: absolute+relative cursor positioning for large displays with a mobile touchscreen. In Proc. UIST '09 (2009), 153-156.

15. Moscovich, T., and Hughes, J. F. Multi-finger cursor techniques. In Proc. GI 'O6 (2006), 1-7.

16. Pietroszek, K., and Lank, E. Clicking blindly: Using spatial correspondence to select targets in multi-device environments. In Proc. MobileHCI'12 (2012), 331-334.

17. Schmidt, D., Block, F., and Gellersen, H. A comparison of direct and indirect multi-touch input for large surfaces. In Proc. INTERACT'09 (2009), 582-594.

18. Sears, A., Revis, D., Swatski, J., Crittenden, R., and Shneiderman, B. Investigating touchscreen typing: The effect of keyboard size on typing speed. Behaviour \& Information Technology 12 (1993), 17-22.

19. Vogel, D., and Baudisch, P. Shift: a technique for operating pen-based interfaces using touch. In Proc. CHI '07 (2007), 657-666.

20. Wang, F., and Ren, X. Empirical evaluation for finger input properties in multi-touch interaction. In Proc. CHI'09 (2009), 1063-1072.

21. Wigdor, D., Shen, C., Forlines, C., and Balakrishnan, R. Effects of display position and control space orientation on user preference and performance. In Proc. CHI 'O6 (2006), 309-318.

22. Wobbrock, J. O., Findlater, L., Gergle, D., and Higgins, J. J. The aligned rank transform for nonparametric factorial analyses using only anova procedures. In Proc. CHI'11 (2011), 143-146. 